\title{
Global Policy on Sexually Transmitted Infections: Frameworks and interventions in Zimbabwe
}

\author{
Hope Taderera \\ Department of Political and Administrative Studies, University of Zimbabwe, P.O Box MP \\ 167, Mount Pleasant, Harare, Zimbabwe \\ Email: htaderera@gmail.com
}

Accepted: October 07, 2012 Published: November 20, 2012

Doi:10.5296/jsr.v3i2.2733 URL: http://dx.doi.org/10.5296/jsr.v3i2.2733

\begin{abstract}
The discussion is on the Global Strategy for the Prevention and Control of Sexually Transmitted Infections (2006-2015), and the Zimbabwe National HIV and AIDS Strategic Plan (2011-2015), a policy intervention ratified towards this end. The Global Strategy was initiated for adoption by national governments to contribute towards Millennium Development Goal number six. It is aimed at providing a framework to guide an accelerated global response for the prevention and control of sexually transmitted infections. STI prevention and control interventions advocated include the promotion of health sexual behavior, provision of condoms and other barrier methods, delivery of prevention and care, strategies for sexual partner notification, provision of medicines, and vaccines, syndromic management and improving information for policy and programme development. The Strategic Plan in Zimbabwe is a multi-sectoral framework developed to provide strategic direction in planning, coordination, implementation, monitoring and evaluation of the national multi-sectoral and decentralized STI, HIV and AIDS interventions. This strategy is being implemented through the Public Sector, Private Medical Sector and the Not for Profit Health Sector. It was concluded that Zimbabwe's policy intervention is in line with the global strategy. From the discussion, it was recommended that Zimbabwe must to systematically build the capacity of STI interventions to contribute effectively towards the realization of Millennium Development Goal Number Six.
\end{abstract}

Key words: Policy, STIs, Global, Zimbabwe 


\section{Introduction}

The current discussion focuses on the global policy strategy on STIs, and on frameworks and interventions in Zimbabwe. Sexually transmitted infections (STIs) are a global challenge which is a major cause of morbidity, mortality and underdevelopment. The World Health Organization's Global Strategy for the Prevention and Control of Sexually Transmitted Infections (2006-2015) is a policy intervention meant to address this challenge, towards the attainment of, in particular, Millennium Development Goal number 6. Zimbabwe has ratified this global strategy through the National HIV and AIDS Strategic Plan (2011-2015). This is a multi-sectoral strategic framework developed to inform and guide the national response to HIV/AIDS and STI related deaths by 2015. In dealing with STIs in this country, the policy strategy is aimed at interrupting transmission, reduce infections and the duration of infection, and prevent the development of complications in STI clients through early diagnosis and syndromic management.

\section{Sexually Transmitted Infections}

Sexually transmitted infections (STI), also referred to as sexually transmitted diseases (STD) and venereal diseases (VD), are illnesses that have a significant probability of transmission between humans by means of human sexual behavior, including vaginal intercourse, oral sex, and anal sex (Blanchard, 2002). While in the past, these illnesses have mostly been referred to as STDs or VD, in recent years the term sexually transmitted infections (STIs) has been preferred, as it has a broader range of meaning a person may be infected, and may potentially infect others, without having a disease. Some STIs can also be transmitted through the use of $\underline{\text { needles }}$ after its use by an infected person, and through childbirth or breastfeeding. An infection is colonization by a parasitic species, which may not cause any adverse effects. In a disease the infection leads to impaired or abnormal function. In either case the condition may not exhibit signs or symptoms (Brunham and Plummer, 1990).

There are more than 30 different sexually transmissible bacteria, viruses and parasites. The most common conditions they cause are gonorrhoea, chlamydial infection, syphilis, trichomoniasis, chancroid, genital herpes, genital warts, human immunodeficiency virus (HIV) infection and hepatitis B infection (World Health Organization, 2007). Several, in particular HIV and syphilis, can also be transmitted from mother to child during pregnancy and childbirth, and through blood products and tissue transfer. Most of the time, people do not know that they are infected with an STI until they are tested or start showing symptoms of disease. Policy strategies have been adopted at a global level to reduce and eliminate the impact of STIs on human and socio economic, reduce related morbidity and mortality, prevent HIV infection, prevent serious complications in women, and to prevent adverse pregnancy outcome (Ibid). 


\section{Global Strategy on STIs}

The Global Strategy for the Prevention and Control of Sexually Transmitted Infections 2006-2015 was developed using an inclusive and broad consultative process within the WHO Secretariat and with WHO Member States and other external partners (National AIDS Council, 2011; World Health Organization, 2007). The process was led by the Controlling Sexually Transmitted and Reproductive Tract Infections team within the Department of Reproductive Health and Research (RHR), which is part of the Family and Community Health (FCH) Cluster at WHO Headquarters in Geneva (World Health Organization, 2011). Developed between September 2002 and March 2006, the global strategy incorporates elements from strategies for the prevention and control of sexually transmitted infections (STIs) developed by WHO regional offices, as well as from consultations held with experts from all WHO regions. It also includes recommendations from members of the WHO Gender Advisory Panel and the Expert Advisory Panel on Sexually Transmitted Infections including those due to Human Immunodeficiency Virus. The strategy complements the global health-sector strategy for HIV/AIDS and WHO's Reproductive Health Strategy to accelerate progress towards the attainment of international development goals and targets. The strategy is implemented in line with MDG number six, target seven which calls on nations to have policy interventions to reverse the spread of HIV/AIDS by 2015.

\subsection{Objectives of the Strategy (2006-2015)}

The purpose of the global strategy is to provide a framework to guide an accelerated global response for the prevention and control of sexually transmitted infections, towards the attainment of international development goals. In particular, the strategy focuses on the achievement of objectives which include increasing the commitment of national governments and national and international development partners for prevention and control of STIs. The objective is also to promote the mobilization of funds and reallocation of resources, taking into account national prioritized results-oriented interventions that ensure aid effectiveness, ownership, harmonization, results and accountability. It is also aimed at ensuring that policies, laws and initiatives related to provision of care are non-stigmatizing and gender-sensitive within the prevailing socio-cultural context, and to harness the strengths and capacities of all partners and institutions in order to scale up and sustain interventions for prevention and control.

\subsection{Strategic components}

The global strategy is guided by a technical, and an advocacy component. The technical component is the global technical strategy adaptable at the country and regional levels, including ways to package and deliver the key programmatic elements of prevention and 
control in a sustainable manner. The strategy draws on lessons learnt and on clearly successful actions that may be reinforced. It also helps in the identification of shortcomings in such key areas which include availability or suitability of health-care services for priority target populations, diagnosis and treatment of asymptomatic infections, the syndromic approach for the management of abnormal vaginal discharge, management of sexually transmitted infections in sexual partners, attitudes of health-care providers and the availability and reliability of data for planning purposes. It also identifies appropriate opportunities for interfacing and integrating with HIV/AIDS and sexual and reproductive health programmes, and for involvement of the private sector. The strategy is also guided by the advocacy component. The advocacy component is aimed at enforcing the global advocacy campaign to raise awareness and mobilize resources worldwide. This campaign is intended to run alongside other interventions such as campaigns for the elimination of congenital syphilis, the control and eradication of curable genital ulcer diseases, and the control of genital herpes and genital human papillomavirus infections.

\subsection{Target audience}

The target audiences for this strategy include managers of national programmes on HIV/AIDS/sexually transmitted infections and sexual and reproductive health, health-sector stakeholders including public-sector and private-sector health-care providers, health ministers, policy-makers and other decision-makers in the health sector. It also includes international agencies and nongovernmental partners, other governmental departments and agencies, and donors.

\subsection{Prevention and control interventions}

In line with this strategy, programmes need to have an understanding of the populations that are at greatest risk, the behaviours or circumstances which put these populations at risk, the best approaches and interventions to break the chain of transmission, and mechanisms to prioritise, scale up and sustain the interventions (Ibid). Targeted interventions should be prioritized according to the needs, feasibility and availability of resources. The populations whose behaviours and vulnerabilities need to be analysed for possible targeted interventions vary between regions and among countries. Those frequently observed to be in need of targeted interventions include sex workers, their clients who also might have sex with their regular partners, mobile populations such as long-distance truck drivers, fishermen, seafarers and migrant workers, who are at increased risk of infection primarily because of their mobility and high-risk sexual contacts and men who have sex with men who have multiple sexual partners and engage in unprotected anal intercourse (Holmes et al., 1999; World Health Organization, 2007). They may also include men who have sex with men and who also have sex with women, substance users, especially those who also sell or exchange sex to support their habit or who have sex with non-users, and incarcerated persons, especially juveniles (Ibid). It was also frequently observed that external and internal refugees and displaced persons, members of the uniformed services, including military and police, tourists, 
women or men who experience sexual and gender based violence, and children and young people on the street, and those who are abused or are orphans, also make up this group (World Health Organization, 2007).

Adolescents are also at special risk of infection with sexually transmitted pathogens, including HIV, because they might not have the information, skills, health care and support they need while going through sexual development (Dehne and Riedner, 2005). Their sexual relations tend to be unplanned and sporadic, and in many cases result from pressure or force or take place in exchange for acceptance or financial gain. Adolescent women in particular are more vulnerable than men for biological, social and economic reasons. In some cultures where adolescents, especially girls, marry at a young age, national programmes need to recognize that the young girls may be at increased risk because the social and biological factors referred to above still apply to them even though they will be regarded as adults by virtue of being married. The prevention and care of these infections, including HIV infection, among young people will require a range of age appropriate interventions from a variety of different sectors (Ibid). The health sector itself will be responsible for a number of such interventions, through a range of health-system partners (World Health Organization, 2007).

\subsubsection{Promoting health sexual behavior}

An effective response to the spread of sexually transmitted infections starts with prevention by providing accurate and explicit information on safer sex, including correct and consistent use of male and female condoms, as well as abstinence, delay in onset of sexual activity, keeping to one sexual partner or reducing the number of sexual partners. In addition to prevention interventions, health-care services must be available to provide early and effective treatment. Communication about sexual behavioural change is part of an integrated, multilevel, interactive process with communities, aimed at developing tailored messages and approaches using a variety of channels. It should be an integral component of prevention efforts and incorporated into care and support activities. It can increase knowledge, stimulate dialogue within the community, promote essential changes in attitude, reduce stigmatization and discrimination, create demand for information and health-care services, advocate appropriate policies and laws, promote interventions for prevention, care and support, and improve skills and self-esteem. Communication channels for sexual behavioural-change messages may be through peer educators and opinion leaders, health talks through institutional or interpersonal networks, and group discussions. Age-appropriate schools-based programmes help in reaching young people who are attending school, but for the out-of-school population other channels, such as peer education, are necessary. Health education about sexually transmitted infections, and counselling of both infected and uninfected people, including voluntary counselling and confidential HIV testing, should be an integral part of any health service for those infections, as the counselling process creates motivation to change sexual behaviour in both infected and uninfected individuals (Ibid).

3.4.2 Providing condoms and other barrier methods 
The male latex condom is the single, most efficient, available technology to reduce the sexual transmission of HIV and other sexually transmitted pathogens (Holmes et al., 2004; World Health Organization, 2007). Condoms should be promoted and distributed through both the public and private sectors, in clinical and non-clinical settings. Maternal and child health and family planning clinics are good additional outlets for condoms, making them accessible to women who could be at risk of sexually transmitted infections. Social marketing programmes have been shown to be particularly effective in ensuring that high-quality, affordable condoms are available where and when they are needed, in both traditional and non-traditional outlets. Condom distribution can also be supplemented by community-based distribution and outreach services to target populations (Ibid).

\subsubsection{Delivering prevention and care}

The aim of delivering care services for people with sexually transmitted infections is to prevent the development of long-term complications and sequelae in people already infected and to prevent the spread of infection to uninfected sexual partners, the fetus or the newborn (World Health Organization, 2007). Sexually transmitted infections programmes should promote accessible, acceptable and effective interventions that offer comprehensive case management of infected persons to prevent further infections and their many complications and long-term sequelae (Ibid).

\subsubsection{Syndromic management}

Traditionally, a presumed sexually transmitted infection has been diagnosed by either clinical appearance alone (which is often inaccurate) or a laboratory-based test, which can be complicated and expensive and commonly delays treatment while test results are awaited (Vuylsteke, 2004). Even if desirable, laboratory based diagnosis is often limited, especially in resource-constrained settings, owing to the cost of maintaining a laboratory and a consistent supply of test kits as well as ensuring quality control (Ibid). Against this background, WHO recommends the syndromic management of sexually transmitted infections in patients presenting with consistently recognized signs and symptoms shown in simple flowcharts that can be used at the primary health-clinic level (World Health Organization, 2007). Syndromic management is based on the identification of a group of symptoms and easily recognized signs associated with infection with well-defined pathogens. Treatment for each syndrome is directed against the main organisms within that geographical setting responsible for the syndrome. The syndromic approach has been shown to be highly effective for the management of urethritis and epididymitis in men and genital ulcers in both men and women, and works well in the management of infants with ophthalmia neonatorum. It should be noted that the syndrome of vaginal discharge is neither specific nor sensitive for predicting gonococcal, chlamydial or other cervical infections; however, if the primary objective is to treat vaginitis attributable, for example, to bacterial vaginosis or trichomoniasis the approach is of benefit and becomes cost effective in all settings. 
Sexually transmitted infections often exist without symptoms, particularly in women. Different strategies are required for the detection and management of these asymptomatic infections. Some of the strategies are case-finding or screening, with enhanced interventions for reaching sexual partners in order to provide case management for a presumptive sexually transmitted infection, and increasing knowledge and awareness of individual risk. Case-finding refers to testing in individuals who seek health care for reasons other than a sexually transmitted infection. A very important application of case-finding is the provision of care for such infections in antenatal clinics and in maternal and child-health and family planning clinics (Ibid).

\subsubsection{Strategies for notification of sexual partners}

Partner notification, which is an integral part of case management, is a process whereby the sexual partners of patients diagnosed with sexually transmitted infections are informed of their exposure to infection so that they may seek consultative screening and treatment (World Health Organization, 1999b; 2007). Partner notification aims to prevent re-infection of the index patient and reduce the spread of infections. Three main approaches which may be followed include the use of third parties (usually health-care personnel) to notify sexual partners, index patients notify their sexual partners, or the patients are supplied with medications to deliver to their sexual partners, and index patients agree to notify their sexual partners, with the understanding that health-care personnel will notify those partners who do not present for treatment within a given time. Epidemiological treatment (treatment for the same infection or syndrome as in the index patient) should be given to all recent sexual partners (Ibid).

\subsubsection{Medicines}

Consistent availability of appropriate medicines is essential for a successful sexually transmitted infection control programme (World Health Organization, 2007). Prompt and effective treatment breaks the chain of transmission and prevents the development of complications and long-term sequelae. A medicine that is appropriate for treatment of a sexually transmitted infection is one that is highly efficacious, that has acceptable toxicity, for which microbial resistance is either unlikely or will be delayed, that is administered orally and preferably as a single dose, and that is not contraindicated for pregnant or breast-feeding women. A two-tier medicines policy, with the provision of less effective medicines at the peripheral health-care level and the most effective (usually more expensive) ones only at a referral level, can result in an unacceptable rate of treatment failures, complications and referrals, bring about drug resistance more rapidly and erode confidence in health services. To ensure a consistent supply of safe and effective medicines, countries need to have a sustainable procurement strategy that ensures a 60-day reserve stock at a minimum (Ibid).

\subsubsection{Diagnostic tests}


Some $80 \%$ to $90 \%$ of the global burden of sexually transmitted infections occurs in the developing world where there is limited or no access to appropriate diagnostics (Mayaud and Mabey 2004). There is a need for the development of rapid diagnostic tests in order to improve the quality of care and diagnosis for patients in resource-limited settings. There is a particularly urgent need for improved diagnostics for these infections in HIV endemic areas, as some of them are important cofactors in the transmission of HIV (World Health Organization, 2007).

\subsubsection{Vaccines}

As immunization of populations at risk is, in general, a highly effective method of controlling infectious diseases, the arguments for searching for effective vaccines against sexually transmitted infection, including HIV infection, are compelling (World Health Organization, 1999a; 2007). Such vaccines would be an important addition to the existing armamentarium of prevention technologies (Ibid). Discussions have been held, under the auspices of WHO, to determine appropriate end point measures for human papillomavirus vaccines and encourage recognition of human papillomavirus infection as a public health problem (Aguado and Pagliusi, 2004). WHO is encouraging countries to consider the benefits of introducing these vaccines in their programmes as well as to explore issues of acceptability and feasibility when it comes to implementation of such vaccination programmes (Ibid).

\subsubsection{Improving information for policy and programme development}

Surveillance of sexually transmitted infections at the national, regional and global levels needs to be enhanced for the purposes of advocacy, programme design, monitoring and evaluation, and patient care (World Health Organization, 1999b; World Health Organization, 2007). The basic components of surveillance that need to be enhanced include case-reporting that disaggregates by age and sex, prevalence assessment and monitoring to identify and track the burden of infection in defined populations, assessment of etiology of infection, antimicrobial resistance monitoring, special studies, and for example assessment of quality of care using mystery clients (Ibid).

Surveillance of sexually transmitted infections is closely linked to and has a special role in second-generation HIV surveillance, the latter includes, in addition to HIV surveillance and AIDS case reporting, behavioural surveillance in order to monitor trends in risk behaviour over time and surveillance of sexually transmitted infections in order to monitor the spread of other such infections in populations at risk for HIV (World Health Organization, 2000). Surveillance of sexually transmitted infections should be closely linked to behavioural surveys, especially to surveys on sexual behaviours, determinants of the epidemiology of such infections and health-care seeking behaviours and their relationship to under detection and underreporting of these infections (World Health Organization, 1999b; 2000). Surveillance is also important in assessing which population groups should receive targeted 
interventions. Periodically, there is a need to perform special studies to focus on other surveillance issues that are not part of the routine case reporting or prevalence assessment. These studies may include investigations for outbreaks of particular infections, such as syphilis, lymphogranuloma venereum and chancroid in certain populations and geographical settings. Timely and reliable data are also required to support programme design, monitoring and management. Prevalence studies in various populations help to assess the distribution of sexually transmitted infections, identify priority target populations and estimate the burden of asymptomatic infections in a community. Trend data are useful to evaluate the effectiveness and impact of control programmes and interventions, and also serve as biological markers of trends in unsafe sexual practices (Ibid).

\subsubsection{Monitoring and evaluation}

Progress of programme implementation needs to be monitored in order to ensure that activities are performed as planned, on time and within budgeted resources, and determine whether the activities are producing the expected outcome or impact (World Health Organization, 2007). There is a lack of data at the implementation level that makes it difficult to measure accurately the effectiveness and cost-effectiveness of various interventions. Such information is important for priority setting, strategic planning and resource allocation. A data collection and analysis process should be established to monitor service delivery, quality of care provided, adequacy of staffing patterns, client response and satisfaction, capital and recurrent programme costs to assess efficiency and cost-effectiveness. Further, it is important that the results of such monitoring, which could be limited to operational research, are linked in a meaningful way with programme implementation. The results should be used to evaluate and improve the ongoing programmes and in the design of new ones. More operational research is needed to examine which interventions work best in particular settings, and research on issues related to women's sexual and reproductive health should be conducted in order to guide the formulation of gender sensitive strategies and interventions (Ibid).

\section{Strategy interface with other programmes and partners}

\subsection{Public health care programmes}

Sexually transmitted infections are implicated in programmes concerned with adolescent health, family planning, women's health, safe motherhood, immunization, child survival and HIV prevention (World Health Organization, 2003; 2007). These programmes are interdependent and strategically should be integrated or have interfaces. These interfaces are indispensable for broadening the coverage of interventions for clients, and reducing missed opportunities for the prevention, detection and treatment of sexually transmitted infections. It should also strengthen the collaboration between the public and private health sectors for better quality and wider coverage (Ibid). 


\subsection{HIV/AIDS}

More recent intervention studies have added information and weight to the sexually transmitted infection/HIV co-factor effect (World Health Organization, 2007). Therefore, programmes on all such infections should establish and maintain strong linkages and complement each other's efforts, given the synergistic interactions between HIV and other sexually transmitted infections and the common elements for prevention of both (Ibid).

\subsection{Sexual and reproductive health}

The interface between services for sexually transmitted infections and sexual and reproductive health is extensive (World Health Organization, 2003; 2007). Both seek to improve the quality of life and, in particular, the sexual and reproductive life of women and men. The two sets of services engage in the key areas of activity which include improving access to services for prevention and control of sexually transmitted infection. As antenatal, maternal and child health, and family planning clinics serve many women of reproductive age, they constitute a network of health facilities that have the potential to expand the reach and coverage for care of sexually transmitted infections to a significant segment of the general female population that would otherwise not be reached through specialized clinics and general curative medical services (Ibid).

\subsection{Adolescent health services}

Most projects to improve sexual and reproductive health for adolescents have focused on sexual health counselling and family planning but have neglected care for sexually transmitted infections among their service-delivery objectives (World Health Organization 2007). Involving parents and young people at the appropriate age of maturity in the planning and implementation of interventions for them is crucial in making an impact on their behaviour. Programmes, including sexual and reproductive health services as part of primary health care, should, at a minimum, institute and provide basic interventions to strengthen surveillance of sexually transmitted infections among adolescents and young people. They should also contribute towards improving the improving the awareness and knowledge of adolescents about sexually transmitted infections and their complications, and how to prevent them, and improve adolescents' access to services.

\subsection{Private Sector}

The private sector or traditional healers and informal providers are frequently the first port of call for patients with sexually transmitted infections, even for those who believe that government health clinics are technically superior. Private providers, whether medically qualified or not, are more acceptable to many people because they are perceived to offer better access and confidentiality, and often have the reputation of being less stigmatizing than public sector facilities. Public policy and interventions should necessarily involve the private 
and informal sectors, and public-private partnerships should be established in the provision of care for sexually transmitted infections. Effective and appropriate regulatory measures should be taken by governments to ensure technical quality and accountability in the private sector services. Governments should explore how to establish formal relations for the promotion of appropriate care with pharmacists' unions, traditional healers' associations and other providers, depending on the setting and prevailing policies, laws and regulations. This should be done in collaboration with the communities themselves (Ibid).

\subsection{Community Involvement}

The involvement of the community in decisions that affect their health is important, and programmes to control sexually transmitted infections need to devise mechanisms for obtaining input from the whole community through appropriate representatives of civil society (World Health Organization, 1999a; 2003; 2007). This can best be achieved by forming partnerships with nongovernmental organizations, faith-based organizations, community based organizations and the private sector. Strategies to engage the community include the provision of information in order to increase community awareness of the problem and increase community demand for interventions and services, holding ongoing consultations with the community, involving the community in the design and implementation of interventions, sharing accountability and responsibility with the community for programme outcomes, involving local political leaders and opinion-makers, including traditional and faith leaders, in advocacy for prevention and care of sexually transmitted infections. Strengthening collaboration with, and capacity of, these organizations is important to ensure that they work more effectively in partnership with governments and others in the prevention and control of sexually transmitted infections (Ibid).

\section{STIs Policy Interventions in Zimbabwe}

Zimbabwe has not had stand alone policy instruments in the fight against STIs. Strategies to fight STIs are to be found within and as part of efforts to fight HIV/AIDS. STI interventions in Zimbabwe are to be found within the Zimbabwe National HIV/AIDS Strategic Plan (ZNASP 2) (2011-2015), a policy instrument of the National HIV/AID Policy of 1999 (National AIDS Council, 2011).

\subsection{Zimbabwe National HIV/AIDS Strategic Plan (ZNASP 2) (2011-2015)}

The Zimbabwe National HIV and AIDS Strategic Plan (ZNASP) is a five-year 2011 to 2015, multi-sectoral framework developed to inform and guide the national response towards achieving zero new infections, zero discrimination and zero AIDS related deaths by 2015 (National AIDS Council, 2011; Zichire, 2012). The ZNASP has been developed to provide a strategic framework that will guide and inform the planning, coordination, implementation, 
monitoring and evaluation of the national multi-sectoral and decentralised STIs, HIV and AIDS response with the aim of achieving zero new infections, zero discrimination and zero AIDS related deaths. It was also developed to articulate national priorities, results and targets that all stakeholders and partners will contribute towards, provide the basis for consolidating strategic partnerships and alliances especially with civil society organisations, public and private sector, and development partners, and establish the basis for Zimbabwe to consolidate its efforts in developing sustainable financing mechanisms for HIV and AIDS response (Ibid).

\subsubsection{The strategic orientation of ZNASP II}

Zimbabwe has adopted human rights, evidence and results based approach in its planning and implementation of the national response (National AIDS Council, 2011). The approach is necessary to ensure that Zimbabwe achieves its aspirations of Zimbabwe of zero new infections, zero discrimination, and zero AIDS related deaths by 2015 .

\subsection{Prevention and control of Sexually Transmitted Infections (STIs)}

In Zimbabwe the strategy in dealing with STIs is to interrupt transmission, reduce infections and the duration of infection, and prevent the development of complications in STI clients through early diagnosis and syndromic management. The ZNASP has articulated primary prevention of STIs that include health education, condom use and abstinence from sex if a partner is infected with an STI. Delivering these services health care workers will be trained to diagnose and treat STIs. STI services will be integrated with other HIV and AIDS, and health care services especially MC, FP and PMTCT. At the same time, health care facilities will be equipped with the necessary diagnostic tools and treatment drugs as well as condoms to effectively treat and manage patients with STIs. Communities will be mobilised and sensitised to adopt health-seeking behaviours for both STIs and HIV. Districts with high prevalence of STI will be prioritised. In line with Guiding Principle 6 of the ZNASP, quality STI care services should be made available and accessible at all levels of the health care delivery system and in all communities.

\subsubsection{Strategies}

The strategies include ensuring the availability of appropriate technical capacity and drugs for effective treatment of STIs in all health facilities, upgrading STI management skills of health personnel at each level including community workers, and strengthening the integration of STI management skills into training curriculum of health personnel at both undergraduate and postgraduate levels. The strategies also include strengthening contact tracing and treatment partners for STIs, and addressing barriers faced by women and young people in seeking treatment for STIs and their complications. These barriers include lack of information, education, stigma and negative cultural norms. The instruments also include improving diagnosis and treatment of STI by developing, implementing and evaluating cost-effective 
management guidelines on STIs and their complications, backed by research and appropriate training. They are also intended to educate the community and especially young people on STI health seeking behavior, facilitate the provision of information to everyone attending any level of, the health system for reproductive or sexual health care about STIs and advise them on their prevention. The strategies are also intended to enhance the relationship between health care providers and patients by undertaking systematic review and research and implementing research results. The instruments also seek to provide information on STIs and related conditions in a gender sensitive and integrated manner, and to ensure that every pregnant woman has access to screening for STIs which are vertically transmittable.

The priority strategies include the acceleration of STI education and awareness in the general population and in particular among the most at risk populations as mobile populations and people engaged in multiple and concurrent partnerships. The priority strategies also include strengthening the procurement and supply of STI drugs, test kits and other consumables, and intensifying training of health workers in STI prevention and management. They also include facilitation of STI surveillance, and strengthening health and community systems to improve partner contact tracing (Ibid).

\subsection{The Health Sector in Zimbabwe}

Health care services in Zimbabwe are provided by the Public Sector, Private Medical Sector and the Not for Profit Sector (Taderera et al, 2012). In the Public Sector, the Ministry of Health and Child Welfare (MoHCW), and Local Authorities, which consist of Rural and Urban District Councils, are the major providers of health services in Zimbabwe. Other providers in this category include the Defence Forces, the Prison Services, the Police, and the Ministry of Education Sports and Culture. The Private for Profit Medical Sector consists of Private Clinics, Traditional Health Practitioners, Private Hospitals and General Practitioners. The Not For Profit Private Sector includes Medical Missions and Non Governmental Organization (Government of Zimbabwe, 2009). These are an avenue through which STI interventions are being pursued (Ibid).

\subsubsection{The Academic and NGO Forum on STIs in Zimbabwe}

The Zimbabwe Community Health Intervention Research Project (ZiCHIRe) is collaborating with the Zimbabwe Ministry of Health and Child Welfare, University of Zimbabwe College of Health Sciences, United States Centre for Disease Control and Prevention in Zimbabwe and the National Institute for Communicable Diseases of the National Health Laboratory of South Africa to facilitate the formulation and reformulation of STI interventions in Zimbabwe (Zichire, 2012). ZiCHIRe is also working with the School of Medicine at the University of California, American STD/HIV Prevention Training Centre and the University of Colorado Denver. Together with these partners, ZiCHIRe is stimulating discourse on the epidemiology, clinical manifestations, management, and prevention of STIs with a focus on clinical practice in Zimbabwe. This discourse contributes towards more effective and efficient implementation 
of clinical interventions on STIs thus contributing towards ZNASP 2 (2011-2015) and the Global Strategy on STIs (2006-2015) (Ibid).

\subsubsection{Development partners in health}

Development partners in Zimbabwe's health sector are a cross section of both multilateral and bilateral institutions, international NGOs, and humanitarian and faith-based organizations (Taderera et al., 2012). They include the European Union (EU), United States Agency for International Development (USAID), the Center for Disease Control (CDC) and the UK's Department for International Development (DFID). The United Nations agencies working in health are the United Nations Population Fund (UNFPA), United Nations Children's Fund (UNICEF), United Nations AIDS (UNAIDS) and United Nations Development Programme (UNDP). Other mechanisms through which Zimbabwe is receiving funding include the Global Fund to Fight AIDS, Tuberculosis and Malaria (GFATM), the Expanded Support Programme (ESP), the consolidated appeal process (CAP), Global Alliance for Vaccines and Immunization (GAVI) and the Health Metrics Network (Ibid). They work in different levels and capacities in collaboration with other health actors in implementing STI interventions in Zimbabwe.

\subsubsection{Community System Strengthening}

In the context of ZNASP II, community systems strengthening focuses on community based systems that strengthen community leadership and governance, community organisation, local resource mobilisation, management to developing community skills in advocacy, monitoring and resource management (National AIDS Council, 2011). The capacity of community-based organisations which include Non Governmental Organizations (NGOs), Faith Based Organizations (FBOs), and support groups is strengthened to provide backstopping support to communities. Community systems strengthening in Zimbabwe revolves around strengthening the enabling social, legal and policy environment at community level, mobilisization and engagement of community and religious leaders, and communication and social mobilisation to support community advocacy work, communication and feedback, and social mobilisation. Capacity will also be built around community networks, linkages, partnerships and coordination. Planning and coordination mechanisms at a district, provincial and national level will also be strengthened. ZNASP II allows community based organisations access to technical assistance, capacity building for local resource mobilisation and adequate resource allocations from central level. The capacity of CBOs, FBOs and NGOs in planning, monitoring and evaluation is also being strengthened through participatory approaches, on community planning, leadership and governance. Accountability and ownership is also being enhanced through training on data collection, analysis and reporting.

\subsubsection{Monitoring and evaluation of STI Programmes in Zimbabwe}


Zimbabwe has developed a national Monitoring and Evaluation ( $\mathrm{M}$ and $\mathrm{E}$ ) Plan used for purposes of tracking the implementation of the ZNASP II. In the short term the M and E Plan is used to establish baselines and to track annual performance of ZNASP II. In the long term the $\mathrm{M}$ and $\mathrm{E}$ Plan facilitates a process of measuring the outcome and impact results. These are articulated in the ZNASP II results framework and the M and E Plan itself. Strategies which have been adopted to strengthen the national $\mathrm{M}$ and $\mathrm{E}$ system include capacity development of human resources for $\mathrm{M}$ and $\mathrm{E}$ at the national, provincial and district levels and in the private sector and civil society organisations. They also include mainstreaming of the national $\mathrm{M}$ and $\mathrm{E}$ results and indicators in other sectors to ensure harmonisation and alignment within $\mathrm{M}$ and $\mathrm{E}$ frameworks in line with the three one principles, development of the $\mathrm{M}$ and $\mathrm{E}$ guide with clearly defined indicators and targets, and strengthening the capacity of stakeholders to use the data in decision making and programming.

\subsubsection{Monitoring ZNASP II}

Monitoring of the ZNASP II will be an on-going activity during the life cycle of ZNASP. The process will involve routine data collection by implementing partners using the standardised data collection tools. Additional monitoring will be premised on desk review of periodical reports submitted by implementing and development partners to the National AIDS Council. Routine monitoring will focus on annual targets, and output results. Overall routine monitoring process will aim at ascertaining whether ZNASP implementation is on track, and making desired progress towards attaining the set targets, whether resources (human, financial and material) are being used efficiently, and whether services are available and being easily accessed by all people especially key populations and most at risk populations. It also involves determining whether the involvement and participation of beneficiary communities, and civil society organisations is sustained at desired levels, the impact of barriers, and determining how the ZNASP II strategies are facilitating capacity development, knowledge and skills transfer, the improvement of service delivery systems, and strategic information management.

\subsubsection{Evaluation of the ZNASP II}

Assessment of the extent to which objectives of the strategic plan (ZNASP II) are met requires an array of periodic reviews and evaluations. These evaluations are critical to collection of specific outcome and impact indicator values as well as evaluating some fundamental attributes of programs such as efficacy, equity, relevance, and appropriateness. The Evaluation component therefore has to be strategically planned for utilizing national surveys and surveillances, project evaluations and other similar researches. ZNASP evaluation will be conducted twice during the life cycle of the strategic plan. The evaluation will assess the extent the strategic plan has or is moving towards the achievement of planned annual targets, outcome and impact results. A mid-term review of the ZNASP II will be conducted in 2013 to assess progress being made in reaching set results and targets. The mid-term evaluation will provide an opportunity to rethink national strategies for scaling up 
the national HIV and AIDS response. The assessment will involve a desk review including routine $\mathrm{M}$ and $\mathrm{E}$ data generated by the national system, key informant interviews, the compilation of any relevant data and information, and the organisation of a review workshop where findings are presented and deliberated on by stakeholders. The priorities and strategies of ZNASP II will be modified as necessary based on emerging evidence. By mid 2015, the end-of-term evaluation of the ZNASP II will be undertaken to inform the development of a successor strategic plan (Ibid).

\section{Conclusions}

It was concluded that the Global Strategy for the Prevention and Control of Sexually Transmitted Infections (2006-2015) was initiated for adoption by national governments to contribute towards MDGs through reductions in STI related morbidity and mortality, prevention of HIV infection, prevention of serious complications in women, and prevention of adverse pregnancy outcome. The objectives of this global strategy are to provide a framework to guide an accelerated global response for the prevention and control of sexually transmitted infections, towards the attainment of international development goals. In particular, the strategy focuses on the achievement of objectives which include increasing the commitment of national governments and national and international development partners for prevention and control of STIs. It was also concluded that the objective is also to promote the mobilization of funds and reallocation of resources, taking into account national prioritized results-oriented interventions that ensure aid effectiveness, ownership, harmonization, results and accountability. It is also aimed at ensuring that policies, laws and initiatives related to provision of care are non-stigmatizing and gender-sensitive within the prevailing socio-cultural context, and to harness the strengths and capacities of all partners and institutions in order to scale up and sustain interventions for prevention and control through a technical and an advocacy component.

The target audiences for this strategy include managers of national programmes on HIV/AIDS/sexually transmitted infections and sexual and reproductive health, health-sector stakeholders including public-sector and private-sector health-care providers, health ministers, policy-makers and other decision-makers in the health sector. It was also concluded that they include international agencies and nongovernmental partners, other governmental departments and agencies, and donors. Through these, prevention and control interventions are pursued, health sexual behaviour is promoted, condoms and other barrier methods are provided, prevention and care are delivered, and strategies for the notification of sexual partners are pursued. The provision of medicines, and vaccines is also provided for. Information for improving programme interventions is enhanced through surveillance, and second-generation HIV surveillance to generate data for advocacy, programme design and monitoring, and monitoring and evaluation. In line with this strategy, it was also concluded that there should also be an interface with other programmes and partners in all sectors of the economy.

It was also concluded that Zimbabwe has not had stand alone policy instruments in the fight 
against STIs. The current strategy to fight STIs is the Zimbabwe National HIV and AIDS Strategic Plan (ZNASP), a five-year 2011 to 2015, multi-sectoral framework developed within the context of the National HIV/AIDS Policy of 1999. The strategy is aimed at dealing with STIs to interrupt transmission, reduce infections and the duration of infection, and prevent the development of complications in STI clients through early diagnosis and syndromic management through health services provided by the Public Sector, Private Medical Sector and the Not for Profit Sector.

From the discussion, it was also concluded that an academic and NGO Forum on STIs in Zimbabwe is also playing an active part. In this, the Zimbabwe Community Health Intervention Research Project (ZiCHIRe) is collaborating with the Zimbabwe Ministry of Health and Child Welfare, University of Zimbabwe College of Health Sciences, United States Centre for Disease Control and Prevention in Zimbabwe and the National Institute for Communicable Diseases of the National Health Laboratory of South Africa to facilitate the formulation and reformulation of STI interventions in Zimbabwe. ZiCHIRe is also working with the School of Medicine at the University of California, American STD/HIV Prevention Training Centre and the University of Colorado Denver. Together with these partners, $\mathrm{ZiCHIRe}$ is stimulating discourse on the epidemiology, clinical manifestations, management, and prevention of STIs with a focus on clinical practice in Zimbabwe. This discourse contributes towards more effective and efficient implementation of clinical interventions on STIs thus contributing towards ZNASP 2 (2011-2015) and the Global Strategy on STIs (2006-2015). Zimbabwe is also working with other development partners drawn from multilateral and bilateral institutions.

In the context of ZNASP II, it was also concluded that community systems strengthening focuses on community based systems that strengthen community leadership and governance, community organisation, local resource mobilisation, management to developing community skills in advocacy, monitoring and resource management. In this, the capacity of community-based organisations which include Non Governmental Organizations (NGOs), Faith Based Organizations (FBOs), and support groups is strengthened to provide backstopping support to communities. It was also concluded that Zimbabwe has developed a national Monitoring and Evaluation ( $\mathrm{M}$ and $\mathrm{E}$ ) Plan used for purposes of tracking the implementation of the ZNASP 2.

\section{Recommendations}

It is recommended that Zimbabwe continues to build its policy capacity in responding to STIs, and contributing towards MDGs. This can be achieved through the systematic approach to policy implementation which is allowing the country to continually improve the design of its STI interventions. From the discussion, it is further recommended that Zimbabwe must develop an STI Strategy separated from HIV/AIDS strategies so that efforts to address the former are not overshadowed by the later. More inquiry on this subject matter is also recommended in 2015, to draw lessons from the implementation of the ZANSP 2 and the 


\section{MInstitute ${ }^{\text {Macrothink }}$}

Global Strategy on Sexually Transmitted Infections, as part of a pedagogic discourse to systematically influence and improve subsequent policy interventions in the global fight against STIs.

\section{Acknowledgements}

This research was made possible by the Faculty of Social Studies, University of Zimbabwe, and ZiCHiRe and its partner organizations including the Ministry of Health and Child Welfare, Zimbabwe.

\section{References}

Blanchard, J.F. (2002): Populations, pathogens, and epidemic phases; closing the gap between theory and practice in the prevention of sexually transmitted diseases. Sexually Transmitted Infections, 78 (Suppl 1): i183-i188.

Brugha, R. Zwi, A.B. (1999): Sexually transmitted disease control in developing countries; the challenge of involving the private sector. Sexually Transmitted Infections, 1999, 75:283285.

Brunham, R.C. Plummer, F.A. (1990): A general model of sexually transmitted diseases and its implication for control. The Medical Clinics of North America, 74:1339-1352.

Dallabetta, G.A. Gerbase, A.C. Holmes, K.K. (1998): Problems, solutions, and challenges in syndromic management of sexually transmitted diseases. Sexually Transmitted Infections, 74 (Suppl 1):S1-S11.

Dehne, K.L. Riedner, G. (2005): Sexually transmitted infections among adolescents: the need for adequate health services. Geneva, World Health Organization and Deutsche Gesellschaft für Technische Zusammenarbeit.

Holmes, K.K. Sparling, P.F. Mardh, P.A. (1999): Sexually transmitted diseases, 3rd Edition. McGraw/Hill, New York.

Mayaud, P. Mabey, D. (2004): Approaches to the control of sexually transmitted infections in developing countries: old problems and modern challenges. Sexually Transmitted Infections, 80:174-182.

National AIDS Council (2011): Zimbabwe National HIV and AIDS Strategic Plan (ZNASP 2) (2011-2015). Government of Zimbabwe Publications, Harare

Pagliusi, S.R. Aguado, M.T. (2004): Efficacy and other milestones for human papillomavirus vaccine introduction. Vaccine, 23:569-578. 


\section{Macrothink}

Journal of Sociological Research

ISSN 1948-5468 2012, Vol. 3, No. 2

Taderera, H. Zhou, G. Madhekeni, A. Chevo, T. (2012): Sector Wide Approach in Health; Policy Response and Framework in Zimbabwe. Journal of Public Administration and Governance, Vol 2 (1) 2012, 158-175.

Vuylsteke, B. (2004): Current status of syndromic management of sexually transmitted infections in developing countries. Sexually Transmitted Infections, 80:333-334.

World Health Organization (1999a): Sexually transmitted diseases: policies and principles for prevention and care. Joint United Nations Programme on HIV/AIDS and World Health Organization, World Health Organization, Geneva.

World Health Organization (1999b): Guidelines for Sexually Transmitted Infections Surveillance. World Health Organization and Joint United Nations Programme on HIV/AIDS. World Health Organization, Geneva.

World Health Organization (2000): Guidelines for second generation HIV surveillance. Joint United Nations Programme on HIV/AIDS and World Health Organization, Geneva.

World Health Organization (2003): Guidelines for the management of sexually transmitted infections. World Health Organization, Geneva.

World Health Organization (2007): Global Strategy for the Prevention and Control of STIs 2005-2015. World Health Organization, Geneva

Zimbabwe Community Health Intervention Research Project 2012: Update on Sexually Transmitted Infections 2012. Harare 